\title{
Atenuación del artefacto metálico post cirugía de columna lumbar mediante protocolo de obtención de imagen en tomografía computarizada multicorte. Estudio de cohortes.
}

\author{
Metal artifact reduction in post-operative spinal imaging using \\ image acquisition protocol in multidetector computed \\ tomography scans. Cohort study
}

\section{L.M. Romero Muñoz, M. Alfonso Olmos, C. Villas Tomé}

\section{RESUMEN}

Fundamento. Las imágenes obtenidas mediante estudios con TC pueden ser alteradas por la presencia de artefactos metálicos, induciendo en ocasiones a malinterpretación en la correcta ubicación de los tornillos pediculares en columna lumbar. El objetivo de este trabajo es desarrollar un protocolo de atenuación del artefacto metálico con el propósito de reducir el artefacto generado por los tornillos pediculares de titanio en aquellos pacientes a los que se ha requerido instrumentación lumbar, permitiendo a los cirujanos de columna realizar un diagnóstico preciso de la ubicación del tornillo pedicular instrumentado.

Material y métodos. En la primera parte del trabajo se realizó un estudio observacional, analítico, longitudinal y retrospectivo en 23 pacientes (103 tornillos pediculares) a los que se requirió artrodesis lumbar por patología degenerativa. En el postoperatorio se les realizó un estudio con TC secuencial evaluando la sobredimensión generada por el artefacto del tornillo, aplicando un protocolo estándar de obtención de imágenes. En la segunda parte del estudio se diseñó un estudio observacional, analítico, longitudinal y prospectivo empleando un equipo de TCMC de 64 coronas aplicando un protocolo específico de adquisición de imagen sobre 18 pacientes (104 tornillos pediculares) intervenidos mediante artrodesis lumbar.

Resultados. En la primera parte del estudio el artefacto medio a ambos lados del tornillo que se genera tras el estudio de TC es de 1,045 mm (DE: 0,45). En el grupo en el que se aplica el protocolo de atenuación del artefacto tras la TCMC la sobredimensión fue de 0,005 en la porción proximal del tornillo y de 0,025 en la parte distal del tornillo.

Conclusiones. Los resultados obtenidos sugieren que tras la aplicación del protocolo de atenuación del artefacto en equipo de TCMC de 64 coronas el artefacto es reducido a unas dimensiones similares a la dimensión real del tornillo pedicular.

Palabras clave. Artefacto. TC. Tornillo. Columna lumbar.

An. Sist. Sanit. Navar. 2015; 38 (3): $417-424$

Departamento de Cirugía Ortopédica y Traumatología. Clínica Universidad de Navarra.

Recepción: 2 de mayo de 2015

Aceptación provisional: 24 de julio de 2015

Aceptación definitiva: 31 de agosto de 2015

\begin{abstract}
Background. In postoperative patients with metallic implants, CT scans can become less effective due to metal-related artifacts. The purpose of our study was to evaluate the effectiveness of a specific metal artifact reduction image protocol, in order to reduce the metal artifact caused by titanium pedicular screws in patients undergoing lumbar pathology by lumbar fusion. This enables surgeons to make an accurate diagnosis of the exact placement of inserted pedicle screws, making this the preferred image modality for assessing screw position after surgery.

Methods. In the first part of the study, CT scans were performed on 23 patients (103 titanium alloy pedicle screws) undergoing a lumbar instrumented fusion for treatment for degenerative disease with a standard image acquisition protocol evaluating the possible overdimension caused by the artifact. In the second part, a prospective study was performed using 64-slice multidetector-row computed tomography (MDCT) on 18 patients (104 titanium alloy pedicle screws) undergoing a lumbar instrumented fusion using a specific image acquisition protocol.

Results. Our results show that in the sequential CT scan group, mean overdimension (on each side) due to brightness was $1.045 \mathrm{~mm}$ (SD 0.45). In the 64-slice multichannel CT group, mean overdimension (on each side) due to brightness was $0.005 \mathrm{~mm}$ at the proximal part of the screw and $0.025 \mathrm{~mm}$ at the distal part of the screw.

Conclusions. The use of a specific metal artifact reduction image protocol in MDCT produces a minimal artifact following lumbar fusion with pedicle screws.
\end{abstract}

Keywords. Artifact. CT scan. Screw. Lumbar spine. 


\section{INTRODUCCIÓN}

El empleo de tornillos pediculares como tratamiento de la patología vertebral se ha incrementado en popularidad en los últimos años $^{1}$. En determinadas ocasiones el cirujano puede evaluar nuevos componentes de dolor y síntomas neurológicos tras una intervención y es por ello que requiere conocer la ubicación exacta del implante con el fin de evitar cirugías de revisión innecesarias $^{2}$. En la actualidad la evaluación de la ubicación de los tornillos pediculares es mediante estudio con tomografía computarizada (TC), dada la baja sensibilidad que presentan los estudios con radiología simple para detectar perforación en el hueso cortical del pedículo vertebral. No obstante a pesar de los avances en la tecnología de la TC, los artefactos provenientes de los implantes metálicos siguen siendo en la actualidad un problema sin solución en la práctica clínica $^{3}$. Estos artefactos afectan frecuentemente estructuras anatómicas, degradando significativamente el valor diagnóstico de la imagen. Diversos autores han demostrado la dificultad en la interpretación en la región de interés alrededor de los implantes en aquellos pacientes que portan implantes ortopédicos metálicos, con los problemas médicos y legales que ello implica ${ }^{4}$.

Determinados autores describen que artefactos localizados hasta $2 \mathrm{~mm}$ dentro del canal raquídeo pueden ser considerados como cuestionables ${ }^{5}$. Es por ello que el conocimiento de la ubicación precisa de los implantes pediculares debe de ser precisa. Las novedades en los equipos de TC de última generación como los equipos de TC multicorte (TCMC) combinadas con las mejoras en las estaciones de trabajo han conseguido reducciones del artefacto metálico. No obstante la TCMC no ha solventado del todo el problema del artefacto, la imagen alrededor del hueso y partes blandas sigue siendo un reto ${ }^{6}$. Se han desarrollado determinados técnicas de supresión del artefacto metálico basados en el preprocesado y en el postprocesado de la imagen. Sin embargo en la literatura revisada y en los estudios analizados el artefacto eran medidos de forma cualitativa y no de forma cuantitativa ${ }^{7}$.
La hipótesis que proponemos en nuestro estudio es que el artefacto que generan los distintos tornillos pediculares instrumentados en columna lumbar tras estudio con tomografía computarizada es medible y cuantificable, pudiéndose conocer la posición exacta de los tornillos pediculares de titanio utilizados en columna lumbar.

Los objetivos del estudio son: cuantificar la sobredimensión que genera el tornillo de titanio tras la realización de una tomografía computarizada con TC secuencial en el postoperatorio de pacientes intervenidos mediante artrodesis lumbar y cuantificar la sobredimensión que genera el tornillo de titanio tras el empleo de protocolo de atenuación de artefacto en TCMC en pacientes intervenidos mediante artrodesis lumbar.

\section{MATERIAL Y MÉTODOS}

Se diseña un estudio clínico de TC de cuantificación del artefacto que generan los tornillos pediculares, tras la aplicación de un protocolo de atenuación del artefacto en adultos voluntarios intervenidos por patología lumbar. El estudio está constituido por dos partes: la primera parte del estudio es un estudio observacional, analítico, longitudinal de cohortes retrospectivo, en la que se evalúa una cohorte de pacientes intervenidos mediante artrodesis posterolateral con tornillos pediculares y posterior realización de estudio de TC secuencial mediante la aplicación de un protocolo estándar de obtención de imágenes. La segunda parte del estudio es un estudio observacional, analítico, longitudinal de cohortes y prospectivo en la que se cuantifica la sobredimensión generada por el tornillo pedicular en una cohorte de pacientes intervenidos por artrodesis lumbar tras el empleo de un protocolo de atenuación del artefacto en TCMC.

La población del estudio constó de 41 pacientes intervenidos mediante artrodesis lumbar con tornillos pediculares, durante el periodo 1997 a 1999 empleando un equipo de TC secuencial y de 2008 a 2010 utilizando TCMC. Los criterios de inclusión fueron: presentar una edad comprendi- 
da entre los 18 y 80 años de edad, fusión posterior con tornillo pedicular de dimensiones conocidas. Se excluyeron aquellos pacientes que habían sido intervenidos en otro centro o que se desconocía el tipo de tornillo pedicular empleado. Asimismo las pacientes embarazadas y los pacientes que una vez informados no aprobaron su inclusión fueron excluidos del estudio.

Para la primera parte del estudio se emplearon un total de 103 tornillos pediculares cilíndricos sobre 23 pacientes que requirieron fusión lumbar posterolateral en nuestro centro. El tornillo presentaba un diámetro de $5,5 \mathrm{~mm}$ y una composición de titanio (Ti6Al4V). Todos los pacientes fueron evaluados en el postoperatorio mediante TC secuencial (SOMATOM HiQ Siemens, Alemania), con los siguientes parámetros de adquisición de imagen: voltaje de tubo de $120 \mathrm{kV}$, corriente de tubo 475 mAs. El espesor de corte empleado fue de $2 \mathrm{~mm}$, con un intervalo de $2 \mathrm{~mm}$, sin solapamiento en las imágenes obtenidas. El kernel empleado fue dirigido a estructuras óseas con un centro constante de $400 \mathrm{UH}$ y una ventana de $2000 \mathrm{UH}$. El field of view incluía todas las vértebras fusionadas para evaluar todos los tornillos pediculares.

La segunda parte del estudio constaba de 104 tornillos pediculares sobre un total de 18 pacientes que requirieron artrodesis posterolateral. Los tornillos empleados eran cónicos con un diámetro de $4,9 \mathrm{~mm}$ en su porción distal y de $7,3 \mathrm{~mm}$ en su porción proximal y estaban compuestos por una aleación de titanio (Ti6Al4V). Todos los pacientes fueron evaluados en el postoperatorio mediante empleo de TCMC de 64 coronas (Somatom Sensation 64, Erlagen, Alemania). El protocolo de obtención de imagen fue $120 \mathrm{kV}, 300 \mathrm{mAs}$ y se utilizó un sistema de modulación angular (CARE dose). La sección de reconstrucción empleada fue de $1 \mathrm{~mm}$ de grosor. Filtro de 60S. Ventana dirigida a estructuras óseas. Colimación 0,6s. Pitch 0,9. Matriz 512x512 (FOV 157x157). La reconstrucción de la imagen se obtuvo a través de la estación de trabajo Leonardo (Siemens) bajo un nivel constante de centro y de ventana (400 y $2000 \mathrm{UH}$ ). Los cortes fueron paralelos al pedículo cada $1 \mathrm{~mm}$. Se seleccionó el corte axial que atravesaba el centro del pedículo vertebral.

Para la cuantificación en la primera parte del estudio se empleó un pie de rey (Vernier) con una precisión de $0,01 \mathrm{~mm}$. Posteriormente se calibró la escala presente en la TC y se medía la dimensión del tornillo de titanio en mm. Para la segunda parte del estudio se cuantificó el diámetro del tornillo en su porción distal y proximal empleando el software que aporta la TC para la medición (Fig. 1).

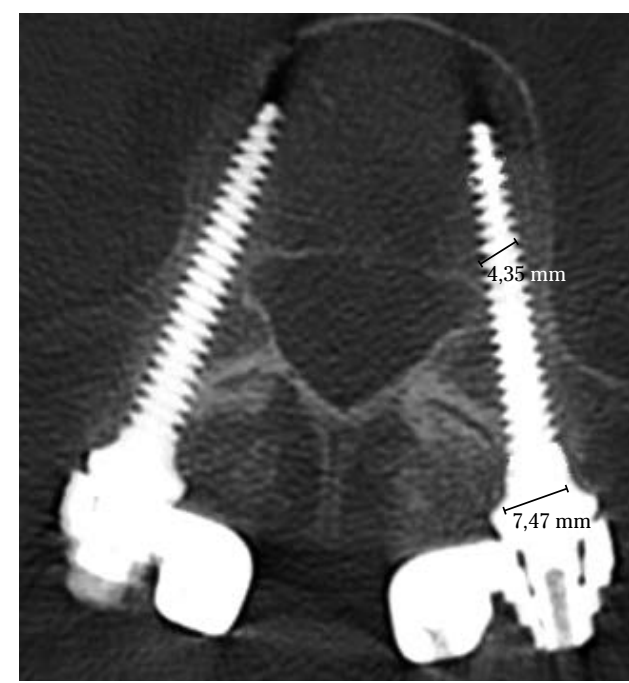

Figura 1. Medición de tornillo L4 izquierdo aplicando el protocolo de obtención de imágenes.

El proyecto ha sido presentado y aprobado por el Comité de Ética de Investigación de la Clínica Universidad de Navarra. En todos los casos se obtuvo el consentimiento informado de los pacientes en el que se manifestaba, de forma expresa, que su inclusión suponía la realización de un estudio de TC a nivel lumbar y sacro siendo empleada una dosis de radiación estudiada por el servicio de radiología de nuestro centro como la dosis mínima y necesaria para poder realizar el estudio. Se realizó una adecuada codificación y disociación identificativa en la base de datos para el correcto mantenimiento de la confidencialidad respecto a la información de los pacientes. 


\section{Análisis estadístico}

El estudio estadístico descriptivo se ha realizado caracterizando las variables continuas mediante estadísticos centrales. Las medidas de tendencia central empleadas para la variable cuantitativa (diámetro del tornillo) son la media aritmética y la mediana. Las medidas de dispersión empleadas son la desviación media, rango, varianza y la desviación típica o estándar.

En relación a la fase analítica con independencia del supuesto de normalidad, se aplicaron pruebas no paramétricas dado que el tamaño muestral del estudio resultaba ser inferior a 30 elementos. Para valorar la correlación existente entre la edad y la dimensión del tornillo tras la TC se realizó un coeficiente de correlación para pruebas no paramétricas (Rho de Spearman). Para evaluar la sobredimensión del artefacto sobre las mediciones de los tornillos se analizaron las mediciones para cada paciente en cada lado y en los diferentes niveles (lumbar y sacro) de posición de los tornillos. Debido a la naturaleza correlacionada de los datos (un mismo paciente puede tener hasta 10 mediciones repetidas) se construyeron diferentes modelos GEE (Ecuaciones de Estimación Generalizada) de la familia gausiana (link de identidad) y una matriz de correlaciones intercambiable al ser la estructura más flexible y adaptada a este tipo de datos. Se comprobó si la edad del paciente, el sexo, el lado y el nivel de posición del tornillo tenían una relación con la magnitud del artefacto (modelo completo). Se redujo el modelo mediante criterios estadísticos (cambios en la verosimilitud) y de control de confusión (cambios en los coeficientes de regresión) hasta llegar a un modelo mínimo simplificado. Se exploró si la edad (en dos grupos, por debajo y por encima de los 40 años) y el sexo actuaban también como posibles modificadores de efecto sobre la magnitud del artefacto relacionada con el lado y nivel de la posición del tornillo (modelo estratificado).

Todas las estimaciones fueron acompañadas de su correspondiente intervalo de confianza del 95\% (IC 95\%).
Todos los cálculos se realizaron con el programa estadísticos SPSS® (Versión 20/ SpSS Inc, Chicago, EEUU).

Para la estimación del tamaño muestral se realizaron los cálculos aplicando el programa EPIDAT siendo el tamaño muestral el suficiente para la realización del estudio.

\section{RESULTADOS}

Tras aplicar los criterios de inclusión y exclusión establecidos, se analizaron un total de 103 tornillos pediculares sobre 23 pacientes intervenidos mediante fusión posterolateral con tornillos cilíndricos (12 varones y 11 mujeres) con una edad media de 41,8 años. La etiología de la indicación de artrodesis posterolateral fue en 14 pacientes por discopatía crónica, 5 por espondilolistesis, 3 por estenosis de canal y en un caso por condrosarcoma.

El artefacto que genera el tornillo en la TC y que sobredimensiona el tamaño real del tornillo cilíndrico de $5,5 \mathrm{~mm}$ tras realizar la TC postoperatoria es de $2,09 \mathrm{~mm}$. La sobredimensión a cada lado del tornillo tras la realización de la TC aplicando el protocolo de obtención de imagen en el tornillo cilíndrico, presenta una media de 1,045 mm. A nivel L5, la dimensión del tornillo tras la TC tiene una media de $7,89 \mathrm{~mm}$ con una desviación típica de $1,03 \mathrm{~mm}$. A nivel S1, la dimensión del tornillo cilíndrico tras la TC es de $7,17 \mathrm{~mm}$ con una desviación típica de $0,49 \mathrm{~mm}$.

El resultado del modelo completo GEE (Tabla 1) refleja que no existe una relación entre el sexo (hombres y mujeres) y la magnitud del artefacto (dimensión del tornillo cilíndrico tras la TC) p: 0,48. La edad tampoco denota una relación con la magnitud del artefacto p: 0,77 . Del mismo modo, tampoco se exponen diferencias estadísticamente significativas al comparar el lado izquierdo o derecho del pedículo vertebral p: 0,74. Los resultados del modelo GEE sí que exhiben diferencias entre los niveles lumbares y sacro p: 0,005. No obstante, no existen diferencias significativas al comparar niveles lumbares entre sí respecto a la dimensión del tornillo cilíndrico tras la TC (p: 0,27). 
Tabla 1. Modelo completo GEE en tornillo cilíndrico

\begin{tabular}{|c|c|c|}
\hline & Beta (IC95\%) & $\mathbf{p}$ \\
\hline Sexo & & 0,48 \\
\hline Mujer (ref) & - & \\
\hline Hombre & $0,157(-0,278$ a 0,590$)$ & \\
\hline Edad & $0,0025(-0,015$ a 0,020$)$ & 0,77 \\
\hline Lado & & 0,74 \\
\hline Izquierdo (ref.) & - & \\
\hline Derecho & $0,043(-0,213$ a 0,300$)$ & \\
\hline \multicolumn{3}{|l|}{ Nivel } \\
\hline L3-L4 (ref) & - & \\
\hline L5 & $0,202(-0,157$ a 0,562$)$ & 0,27 \\
\hline S1 & $-0,560(-0,953$ a $-0,166)$ & 0,005 \\
\hline Constante & 7,475 & \\
\hline
\end{tabular}

Modelo de estimación GEE: link identidad; matriz de correlaciones intercambiable.

En la segunda parte del estudio cumplieron los criterios de inclusión del estudio 18 pacientes (12 varones y 6 mujeres) intervenidos mediante fusión posterior con tornillos cónicos, obteniendo 104 tornillos pediculares. La indicación de artrodesis posterolateral fue en 14 pacientes por discopatía crónica, 3 pacientes por estenosis de canal y un caso por espondilolistesis. La edad media que presentaban los pacientes fue de 57 años.
La media aritmética de dimensión del tornillo tras la realización de la TCMC con el protocolo de reducción del artefacto en su diámetro distal es de $4,95 \mathrm{~mm}$ con un IC de 4,92 a 4,97 mm y una desviación típica de 0,14 . Respecto al diámetro proximal la media de dimensión del tornillo cónico fue de 7,41 mm con un IC de 7,38 a 7,43 $\mathrm{mm}$ y una desviación típica de 0,13 (Tabla 2).

Tabla 2. Medidas del diámetro distal y proximal del tornillo cónico tras la realización de la TCMC

\begin{tabular}{cccccc}
\hline \multirow{2}{*}{ DIM distal } & Sexo & $\mathbf{N}$ & Media & Desviación típica & Error típico de la media \\
\cline { 2 - 6 } & Mujer & 36 & 4,92 & 0,12 & 0,02 \\
\hline \multirow{2}{*}{ DIM prox } & Hombre & 68 & 4,97 & 0,15 & 0,02 \\
\cline { 2 - 6 } & Mujer & 36 & 7,39 & 0,09 & 0,01 \\
\hline & Hombre & 68 & 7,42 & 0,14 & 0,02 \\
\hline
\end{tabular}

El resultado del modelo completo GEE (Tabla 3) refleja que no existe una relación entre el sexo (hombres y mujeres) y la magnitud del artefacto (dimensión del tornillo cónico tras la TCMC) en la medición del diámetro proximal (p: 0,39) y en la medición del diámetro distal del tornillo cónico (p: 0,24). La edad tampoco muestra una relación con la magnitud del artefacto en su diámetro proximal (p: 0,29$)$ ni en su diámetro distal (p: 0,77). Del mismo modo, tampoco se muestran diferencias estadísticamente significativas al comparar el lado izquierdo o derecho del pedículo vertebral en el diámetro proximal ( $p: 0,63)$ ni en su diámetro distal (p: 0,14). Los resultados del modelo GEE no muestran diferencias al comparar niveles lumbares entre sí respecto al diámetro distal (p: 0,52) ni al comparar nivel lumbar y sacro (p: 0,16$)$. Sí existen di- 
ferencias significativas al comparar niveles lumbares entre sí respecto a la dimensión del tornillo cónico en su diámetro proximal tras la TCMC (p: 0,03). No se observan di- ferencias significativas al comparar nivel lumbar y sacro respecto al diámetro proximal (p: 0,63) (Fig. 2).

Tabla 3. Modelo completo GEE en tornillo cónico

\begin{tabular}{|c|c|c|c|c|}
\hline & $\begin{array}{l}\text { Diámetro distal } \\
\text { Beta (IC 95\%) }\end{array}$ & $\mathbf{p}$ & $\begin{array}{l}\text { Diámetro proximal } \\
\text { Beta (IC 95\%) }\end{array}$ & $\mathbf{p}$ \\
\hline Sexo & & 0,24 & & 0,39 \\
\hline Mujer (ref) & - & & - & \\
\hline Hombre & $0,054(-0,037$ a 0,146$)$ & & $0,031(-0,040$ a 0,102$)$ & \\
\hline Edad & $0,0006(-0,003$ a 0,004$)$ & 0,77 & $0,0016(-0,001$ a 0,005$)$ & 0,29 \\
\hline Lado & & 0,14 & & 0,63 \\
\hline Izquierdo (ref.) & - & & - & \\
\hline Derecho & $0,032(-0,010$ a 0,074$)$ & & $-0,010(-0,052$ a 0,031$)$ & \\
\hline \multicolumn{5}{|l|}{ Nivel } \\
\hline L2-L4 (ref) & - & & - & \\
\hline L4-L5 & $-0,031(-0,123$ a 0,062$)$ & 0,52 & $0,097(0,008$ a 0,186$)$ & 0,03 \\
\hline S1 & $-0,081(-0,177$ a 0,016$)$ & 0,16 & $0,042(-0,051$ a 0,014$)$ & 0,63 \\
\hline Constante & 4,906 & & 7,232 & \\
\hline
\end{tabular}

Modelo de estimación GEE: link identidad; matriz de correlaciones intercambiable.

Figura 2. Grado de artefacto relativo para el nivel sacro (referencia: nivel L2-L3) en diferentes estratos definidos por el género y la edad del paciente).

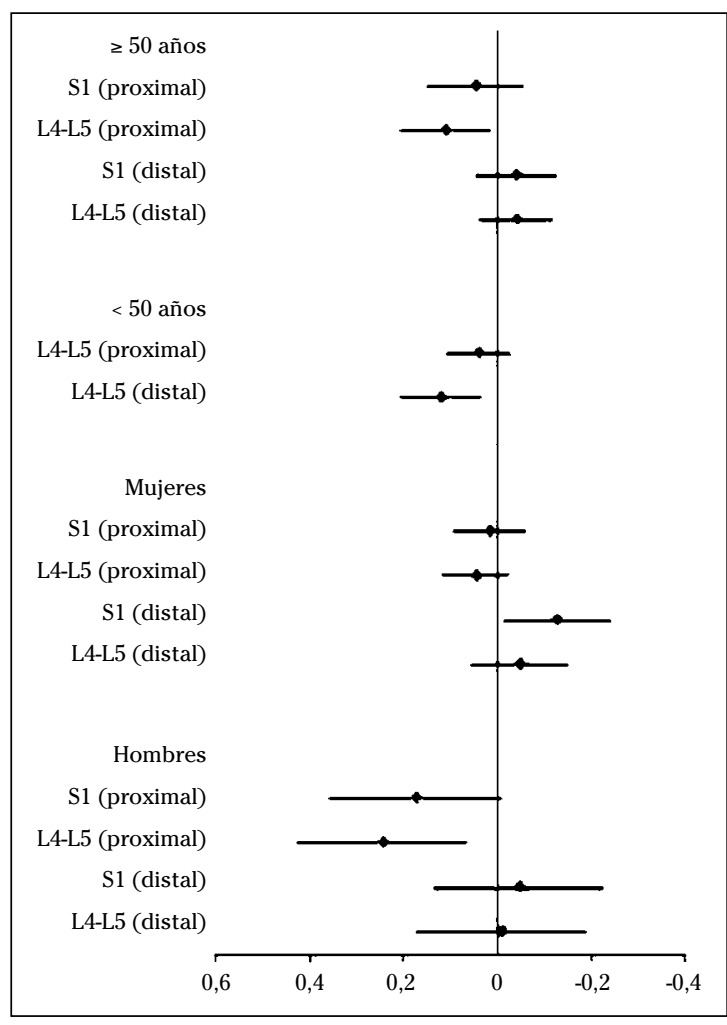

An. Sist. Sanit. Navar. 2015, Vol. 38, № 3, septiembre-diciembre 


\section{DISCUSIÓN}

Hemos estudiado por primera vez a una población española a la que se ha implantado tornillos pediculares en columna lumbar y posteriormente se le ha realizado una TC empleando un protocolo de atenuación de artefacto en TC con el fin de reducir el artefacto que genera el tornillo y se ha constatado una reducción del artefacto en la TC con dos tipos distintos de tornillo pedicular empleando un protocolo diseñado para la atenuación del artefacto. Los resultados obtenidos en el estudio reflejan que aplicando el protocolo de obtención de imágenes con TCMC de 64 coronas la dimensión del tornillo en el estudio con TC es igual a la dimensión real del tornillo pedicular, mostrando que ni el sexo, edad, lado o nivel estudiada muestra diferencias significativas. En relación con el empleo de la TC secuencial se objetivan diferencias estadísticamente significativas al compara niveles lumbares y sacro p:0,005. Estas diferencias obtenidas pueden ser secundarias a la distinta distribución de grasa corporal existente en niveles lumbares y sacro, la grasa corporal puede ser un elemento que influya en la generación de artefactos. Es por ello que en nivel sacro exista una menor proporción de grasa corporal y que sea ese el motivo por el que puedan obtenerse artefactos con una menor dimensión.

De la revisión bibliográfica realizada con razón de tornillos pediculares y artefacto generado en la TC, hay escasas referencias en las que se haya valorado con precisión (medición cuantitativa) el artefacto que genera el tornillo pedicular en la TC. Los trabajos que más se aproximan ${ }^{8-10}$ realizan estudios con distintos protocolos de $\mathrm{kV}$ y mAs en TC y son en su gran mayoría estudios cualitativos, que se basan en valorar si disminuye o no mediante distintos observadores, sin que haya un estudio en la actualidad que haya podido reducir el artefacto del tornillo en la TC a 0.

Haramati y col valoran en su estudio de TC sobre 3 pacientes a los que se valoran dos tipos de implante uno con titanio $\mathrm{y}$ otro con cromo-cobalto ${ }^{11}$. En su estudio cualitativo muestran que no encuentran ventaja al empleo de mayores $\mathrm{kV}$ de cara a reducir el artefacto en la TC. No obstante como crítica a su estudio, emplean grosores de corte de $5 \mathrm{~mm}$ y utilizan una TC helicoidal y no especifican el pitch que emplean. Stradiotti y col comparan utilizando kilovoltajes entre 120 y 140, demostrando que a mayor kilovoltaje mayor penetrancia del rayo dentro del tejido y mayor atenuación del artefacto ${ }^{7}$. Asimismo Moon y col comparan la variación al aplicar valores de kilovoltaje $(120 \mathrm{kV}$ y $140 \mathrm{kV})$ en 3 fémures de espécimen porcino a los que les coloca una placa de acero ${ }^{9}$. Sus resultados revelan que la reducción del artefacto fue estadísticamente significativa incrementando el kilovoltaje $(\mathrm{p}<0,001)$ y reduciendo el grosor de corte en el estudio (p: 0,47) pero resulta no significativa si se aumenta el miliamperaje (p: 0,59). Se observa que el volumen del artefacto fue el mayor cuando empleamos $120 \mathrm{kV}, 100 \mathrm{mAs}$ y $2 \mathrm{~mm}$ de espesor de corte y el mínimo artefacto al emplear $140 \mathrm{kV}, 500 \mathrm{mAs}$ y $0,75 \mathrm{~mm}$ de espesor de corte. Se aprecia cómo con parámetros de referencia de $120 \mathrm{kV}, 100 \mathrm{mAs}$ y $2 \mathrm{~mm}$ de espesor de corte se reduce en un $22 \%$ artefacto que se genera en la TC pasando de $120 \mathrm{kV}$ a $140 \mathrm{kV}$, sin embargo solo se reduce en un $11 \%$ cuando se incrementa el mAs a 1000.

Nuestros resultados respecto a la atenuación del artefacto en la TC se deben en primer lugar al equipo de TCMC empleado en el estudio. La TCMC de 64 coronas es un equipo de última generación de TC con una serie de ventajas respecto a otros TC previos. Una de esas ventajas es la posibilidad de la modulación angular (CARE dose) que incorpora el equipo. La ventaja radica en que los mAs empleados en el estudio dependen de cada paciente y no se emplean unos mAs fijos para el estudio. Esto hace que se reduzca la radiación empleada en el estudio de una manera considerable, en algunas series de hasta un $45 \%{ }^{12}$ ya que determinados pacientes requerirán más radiación por su morfología que otros ${ }^{13}$.

En segundo lugar, el material empleado (Ti6Al4V) en el estudio es el material que menos artefactos genera tras estudio de TC. El empleo de otras aleaciones hubie- 
ra alterado más el artefacto que se genera tras la $\mathrm{TC}^{14}$.

La principal limitación de nuestro estudio radica en que durante el desarrollo no hemos tenido ningún caso de mal posición de tornillos pediculares en columna lumbar. Por lo tanto, al no haber tenido casos de mal posición tanto de tornillos cilíndricos como cónicos, nuestro protocolo no ha sido fundamental para tomar una decisión de reintervenir a un paciente por dolor radicular tras una fusión lumbar con tornillos pediculares. No obstante, creemos que en el supuesto caso, la clínica del paciente y la atenuación del artefacto de los tornillos pediculares con el nuevo protocolo de TC como han mostrado nuestros resultados, hubiera ayudado en la toma de decisiones.

Otra de las limitaciones del estudio es el no haber empleado otros instrumentales que se emplean rutinariamente en cirugía de columna como las barras de distracción o cerclajes sublaminares, de manera que no sabemos el comportamiento de estos instrumentales con el protocolo de atenuación del artefacto con la TCMC.

Creemos que son necesarias futuras investigaciones con el objetivo de optimizar el protocolo de atenuación del artefacto en la TCMC así como aportar el comportamiento de los distintos tipos de tornillos pediculares en la TCMC.

\section{BIBLIOGRAFÍA}

1. Weinstein JN, LuRIE JD, Olson PR. United States' trends and regional variations in lumbar spine surgery: 1992-2003. Spine 2006; 31 : 2707-2714.

2. Mohanty SP, Bhat SN, Ishwara-Keerthi C. The effect of posterior instrumentation of the spine on canal dimensions and neurological recovery in thoracolumbar and lumbar burst fractures. Musculoskelet Surg 2011; 95 : 101-106.

3. Kwon H, Kim KS, Chun YM, Wu HG, Carlson JN, $\mathrm{PARK}_{\mathrm{A}} \mathrm{JM}$ et al. Evaluation of a commercial orthopedic metal artifact reduction tool in radiation therapy of head and neck patients. Br J Radiol 2015; 20: 201-212.

4. Jeong S1, Kim SH, Hwang EJ, Shin CI, Han JK, Сног BI. Usefulness of a metal artifact reduction algorithm for orthopedic implants in abdominal CT: phantom and clinical study results. Am J Roentgenol 2015; 204: 307-317.

5. GERTZBEIn SD1, Robbins SE. Accuracy of pedicular screw placement in vivo. Spine 1990; 15: 11-14.

6. Wallace AB, Goergen SK, Schick D. Multidetector CT dose: clinical practice improvement strategies from a successful optimization program. J Am Coll Radiol 2010; 7: 614-624.

7. Stradiotti P, Curti A, Castellazi G, Zerbi A. Metal-related artifacts in instrumented spine. Techniques for reducing artifacts in CT and MRI: state of the art. Eur Spine J 2009; 18 suppl 1: 102-108.

8. LeE S, Kiм HJ, Сног BK. A pragmatic protocol for reduction in the metal artifact and radiation dose in multislice computed tomography of the spine: cadaveric evaluation after cervical pedicle screw placement. J Comput Assist Tomogr 2007; 31: 635-641.

9. Moon SG, Hong SH, CHOI JY. Metal artifact reduction by the alteration of technical factors in multidetector computed tomography: a 3-dimensional quantitative assessment. J Comput Assist Tomogr 2006; 32: 630-633.

10. Elliott MJ, Slakey JB. CT provides precise size assessment of implanted titanium alloy pedicle screws. Clin Orthop Relat Res 2014; 472: 1605-1609.

11. Haramati N, Staron RB, Mazel-Sperling K. CT scans through metal scanning technique versus hardware composition. Comput Med Imaging Graph 1994; 18: 429-434.

12. Wang Y, Qian B, Li B, Qin G, Zhou Z, Qiu Y et al. Metal artifacts reduction using monochromatic images from spectral CT: evaluation of pediclescrews in patients with scoliosis. Eur J Radiol 2013; 82: 360-366.

13. Liu PT, Pavlicek WP, Peter MB, Spangehl MJ, RoBERTS CC, PADEN RG. Metal artifact reduction image reconstruction algorithm for CT of implanted metal orthopedic devices: a work in progress. Skeletal Radiol 2009; 38: 797-802.

14. Li JY, Pow EH, Zheng LW, Ma L, Kwong DL, CHeung LK. Quantitative analysis of titaniuminduced artifacts and correlated factors during micro-CT scanning. Clin Oral Implants Res 2014; 25: 506-510. 